\title{
ON PARALLELISM IN RIEMANNIAN MANIFOLDS
}

\author{
BY ALAN B. PORITZ ${ }^{1}$
}

\section{Communicated by Philip Hartman, December 15, 1969}

The definition of parallelism along a curve in a Riemannian manifold extends to higher dimensional submanifolds. This note is to announce a local existence and uniqueness theorem, Theorem $\mathrm{B}(\mathrm{p})$, for the extended definition. A proof of the theorem in the $C^{\infty}$ category will appear in [2]. A proof, in the $C^{\omega}$ category, under somewhat weaker conditions, will appear in [1]. A global $C^{\infty}$ version under stronger assumptions appears in [3]. This note ends with a sketch of a new proof of Theorem $B(p)$.

Let $g: N^{p} \rightarrow M^{m}$ be a (not necessarily isometric) smooth (that is, $C^{\infty}$ or $\left.C^{\omega}\right)$ immersion of Riemannian manifolds. Let $E$ be a euclidean vector bundle over $N$ and $F$ a euclidean vector bundle over $M$. A vector bundle map $G: E \rightarrow F$ is a vector bundle isometry along $g$ provided that $G$ sends the fibers $E(n)$ isometrically into the fibers $F(g(n))$. When $E$ and $F$ are the tangent bundles $\left(T\left(N^{p}\right)\right.$ and $\left.T\left(M^{m}\right)\right), G$ is called a tangent bundle isometry (T.B.I.) along $g$. The normal bundle to $a$ T.B.I. $G$ is the $m-p$ dimensional vector bundle $G^{\perp}$ over $N$ whose fiber over $n \in N$ is the orthogonal complement $\perp G\left(N_{n}\right)$ to $G\left(N_{n}\right)$ in $M_{o(n)}$. The second fundamental form of $G$, II $_{G}: G^{\perp} \rightarrow$ $\operatorname{Hom}(T(N), T(N))$ is a vector bundle map defined as follows. Given $v \in \perp G\left(N_{n}\right)$ and $x, y \in N_{n}$ extend $y$ to a vector field $Y$ on $N$ in some neighborhood of $n$, let $\nabla$ be the covariant derivation on $M$ and put

$$
\left\langle\mathrm{II}_{G}(v) x, y\right\rangle_{n}=-\left\langle\nabla_{T_{\theta}(x)} G(Y), v\right\rangle_{o(n)} .
$$

The definition is independent of the choice of $Y$.

$G$ is parallel along $g$ if (trace) $\cdot \mathrm{II}_{G}: G^{\perp} \rightarrow R$ vanishes identically. It was shown in [1] that this definition is a generalization to higher dimensional immersed submanifolds, of the classical notion of parallelism along a curve. The significant facts are the following.

Every unit vector field along a curve $g: N^{1}=(a, b) \rightarrow M$ corresponds in a natural way to a T.B.I. along $g$. Under this correspondence, parallel vector fields are paired with parallel T.B.I.'s.

An immersion $g: N^{p} \rightarrow M^{m}$ is isometric if and only if its tangent map

AMS Subject Classifications. Primary 5372, 5304, 5370, 5374, 3503; Secondary $3596,5730,5720$.

Key Words and Phrases. Parallelism, least area variational problem, minimal immersion, vector bundle isometry, parallel tangent bundle isometry, second fundamental form, normal bundle, Cauchy-Kowalewski Theorem.

1 Partial support by NSF Contract GP-4503 was received during the preparation of this paper. 
$T g: T N \rightarrow T M$ is a T.B.I. In such a situation, $g$ is a minimal immersion if and only if $T g$ is parallel along $g$. Thus for every $p, 1 \leqq p<m$, the critical manifolds of the calculus of variations problem for minimal $p$ dimensional "area" are exactly the $p$ dimensional autoparallels (i.e. the isometric immersions whose tangent maps are parallel).

Below, the same letter is used to designate a distribution on a manifold and the subbundle of the tangent bundle that it determines. If $E$ is a vector bundle over $Y$ and $i: X \rightarrow Y$ is a smooth map then $i_{*}: i^{*} E \rightarrow E$ is the induced map of the induced bundle.

TheOREM $\mathrm{B}(\mathrm{p})$. Let $\mathrm{g}: N^{p} \rightarrow M^{m}$ be an (not necessarily isometric) immersion of Riemannian manifolds. Let $H$ be a $(p-1)$ dimensional distribution on $N^{p}$ and $\left(N^{p-1}, i\right)$ a homeomorphically embedded integral manifold of $H$. Suppose there is given as initial data:

1. $G^{p-1}: H \rightarrow T(M)$, a vector bundle isometry along $g$, and

2. $G^{p}: i^{*} T\left(N^{p}\right) \rightarrow T(M)$, a vector bundle isometry along $g \cdot i$.

It is assumed that $G^{p-1}$ and $G^{p}$ are compatible:

$$
\left.G^{p}\right|_{i * H}=G^{p-1} \cdot i_{*}: i^{*} H \rightarrow T(M) .
$$

Then, if the data is all $C^{\omega}$, there is a neighborhood $U$ of $N^{p-1}$ in $N^{p}$ and a unique parallel $C^{\omega}$ T.B.I. $G: T(U) \rightarrow T(M)$ that extends the initial data:

$$
\begin{aligned}
\left.G\right|_{H} & =G^{p-1}: H \rightarrow T(M) \text { along }\left.g\right|_{U} \quad \text { and } \\
G \cdot i_{*} & =G^{p}: i^{*} T\left(N^{p}\right) \rightarrow T(M) \text { along } g \cdot i .
\end{aligned}
$$

Theorem $B(p)$ is a local extension of the classical theorem that asserts the existence and uniqueness of a parallel unit vector field along a curve $g: N^{1}=(a, b) \rightarrow M$ in terms of initial data at a point $N^{0} \in N^{1}$. In [1], a procedure is developed that proves Theorem $\mathrm{B}(\mathrm{p})$ and at the same time the classical theorem on the local existence and uniqueness of a $C^{\omega}$ minimal immersion in terms of initial data on a codimension one submanifold. The procedure makes use of certain differential forms on the $p$ plane bundles over $N$ and $M$. The solutions of both problems appear as integral manifolds that pass through the initial data. Their existence and uniqueness is a consequence of the CartanKahler Theorem. Here, we sketch a proof of Theorem B(p) using the Cauchy-Kowalewski Theorem directly.

Let $\tilde{n} \in N^{p-1}$. The assumptions on $N^{p-1}$ and $H$ imply the existence of a coordinate neighborhood $\left(V, z_{1}, \cdots, z_{p}\right)$ of $\tilde{n}$ in $N^{p}$ where $\left\|\partial / \partial z_{p}\right\| \equiv 1, N^{p-1} \cap V$ is the slice $z_{p}=0$ and the integral manifolds of the distribution $\perp H$ are the slices $z_{i}=$ constant $i=1, \cdots, p-1$. Because of the compatibility condition on $G^{p-1}$ and $G^{p}$ it may also be assumed that there are fields of orthonormal frames 
$\left\{Z_{1}, \cdots, Z_{p}=\partial / \partial z_{p}\right\}$ on $V$ and $\left\{Y_{1}, \cdots, Y_{m}\right\}$ along $\left.g\right|_{v}$ with the property that a $C^{\omega}$ T.B.I. $G$ defined along $\left.g\right|_{v}$ extends the initial data along $\left.g\right|_{v}$ if and only if its matrix representation $\left(r_{k i}\right)$ with respect to these frames $\left(G\left(Z_{i}\right)=\sum_{k} r_{k i}, Y_{k} i=1, \cdots, p\right)$ satisfies the equations

$$
\begin{aligned}
& r_{k i}=\delta^{k i}, \quad k=1, \cdots, m, \quad i=1, \cdots, p-1, \\
& r_{1 p}=, \cdots,=r_{p-1 p}=0
\end{aligned}
$$

on $V$

and

$$
r_{p p}=1, \quad r_{p+1 p}=, \cdots,=r_{m p}=0 \quad \text { on } V \cap N^{p-1} .
$$

It follows that the T.B.I.'s $G$ that extend the initial data on $V$ are in bijective correspondence with the $m-p$ tuples $\left(r_{p+1 p}, \cdots, r_{m p}\right)$ of $C^{\omega}$ functions on $V$ that vanish on $N^{p-1} \cap V$. The condition that $G$ be parallel along $\left.g\right|_{\nabla}$ is expressed by the vanishing, for each $n \in V$, of the projection of $\sum_{i=1}^{p} \nabla_{Z_{i}(n)} G\left(Z_{i}\right)$ into $\perp G\left(N_{n}^{p}\right)$. On some, perhaps smaller, neighborhood of $\tilde{n}$ this condition is equivalent to the CauchyKowalewski system:

$$
\begin{aligned}
0 & =\left\langle\sum_{i=1}^{p} \nabla_{Z_{i}(n)} G\left(Z_{i}\right), Y_{j}(n)\right\rangle \\
& =\left\langle\sum_{i=1}^{p-1} \nabla_{Z_{i}(n)} Y_{i}, Y_{j}(n)\right\rangle+\sum_{l=p}^{m} r_{l p}\left\langle\nabla_{Z_{p}(n)} Y_{l}, Y_{j}(n)\right\rangle+\frac{\partial r_{j p}}{\partial z_{p}}(n), \\
j & =p+1, \cdots, m .
\end{aligned}
$$

Thus, on some sufficiently small neighborhood $V^{n}$ of any point $\tilde{n} \in N^{p-1}$, there is a unique $C^{\omega}$ parallel T.B.I. $G^{\tilde{n}}$ that extends the initial data along $g \mid v^{n}$. A neighborhood $U$ of $N^{p-1}$ in $N^{p}$ can then be constructed on which there is a unique $C^{\omega}$ parallel T.B.I. $G$ that extends the initial data along $\left.g\right|_{U}$ so that for each $\tilde{n} \in N^{p-1}:\left.G\right|_{U n \nabla^{n}}$ $=\left.G^{n}\right|_{\text {UnV }}$.

\section{BIBLIOGRAPHY}

1. A. Poritz, A generalization of parallelism in Riemannian geometry; The $C^{\omega}$ case, Trans. Amer. Math. Soc. (to appear).

2. - A generalization of parallelism in Riemannian geometry; The $C^{\infty}$ case, J. Differential Geometry (to appear).

3. - A global existence and uniqueness theorem for generalized parallelism, Global Analysis, Proc. Sympos. Pure Math., vol. 15, Amer. Math. Soc., Providence, R. I., 1968, pp. 293-298.

University of Pennsyluania, Philadelphia, Pennsylvania 19104 